\title{
High rate misidentification of biochemically determined Streptococcus isolates from swine clinical specimens
}

\author{
Nattakan MEEKHANON ${ }^{1)}$, Sarawan KAEWMONGKOL ${ }^{11}$, \\ Pichai JIRAWATTANAPONG ${ }^{2}$, Tanyanant KAMINSONSAKUL ${ }^{3)}$, \\ Siriporn KONGSOI ${ }^{4)}$, Suksan $\mathrm{CHUMSING}^{4)}$, Masatoshi OKURA ${ }^{5)}$, \\ Yuichi UENO ${ }^{5)}$, Tsutomu SEKIZAKI ${ }^{6)}$ and Daisuke TAKAMATSU ${ }^{5,7) *}$ \\ 1)Department of Veterinary Technology, Faculty of Veterinary Technology, Kasetsart University, \\ Bangkok 10900, Thailand \\ ${ }^{2)}$ Department of Farm Resources and Production Medicines, Faculty of Veterinary Medicine, \\ Kasetsart University, Nakhon Pathom 73140, Thailand \\ ${ }^{3}$ Kamphaengsaen Veterinary Diagnostic Unit, Faculty of Veterinary Medicine, Kasetsart University, \\ Nakhon Pathom 73140, Thailand \\ 4)Department of Veterinary Public Health, Faculty of Veterinary Medicine, Kasetsart University, \\ Nakhon Pathom 73140, Thailand \\ ${ }^{5)}$ Division of Bacterial and Parasitic Disease, National Institute of Animal Health, National Agriculture and Food \\ Research Organization, Tsukuba, Ibaraki 305-0856, Japan \\ 6) Research Center for Food Safety, Graduate School of Agricultural and Life Sciences, The University of Tokyo, \\ Tokyo 113-8657, Japan \\ 7)The United Graduate School of Veterinary Sciences, Gifu University, Gifu, Gifu 501-1193, Japan
}

J. Vet. Med. Sci.

81(4): 567-572, 2019

doi: 10.1292/jvms.18-0678

Received: 15 November 2018 Accepted: 18 February 2019 Published online in J-STAGE: 27 February 2019
ABSTRACT. In this study, 22 bacterial isolates from swine necropsy specimens, which were biochemically identified as Streptococcus suis and other Streptococcus species, were re-examined using species-specific PCR for authentic S. suis and 16S rRNA gene sequencing for the verification of the former judge. Identification of S. suis on the basis of biochemical characteristics showed high false-positive (70.6\%) and false-negative (60\%) rates. The authentic S. suis showed various capsular polysaccharide synthesis gene types, including type 2 that often isolated from human cases. Five of 22 isolates did not even belong to the genus Streptococcus. These results suggested that the misidentification of the causative pathogen in routine veterinary diagnosis could be a substantial obstacle for the control of emerging infectious diseases.

KEY WORDS: diagnosis, identification, Streptococcus, Streptococcus suis, swine

In addition to viral infection, which greatly affects swine production, bacterial infection, particularly streptococcal infection, is considered as a major problem in the swine industry [23]. Among pathogenic streptococci, Streptococcus suis is the most common cause of septicemia, pneumonia, arthritis and meningitis in piglets [7]. Moreover, because S. suis can be transmitted to humans and leads to serious clinical consequences including meningitis, septic shock and permanent deafness [13], it is considered as the public health threat in many regions, especially in Asian countries. Besides S. suis, other Streptococcus or Streptococcus-like species may considerably affect swine production $[3,10]$.

Although molecular biological approaches have been applied for bacterial identification, the traditional biochemical tests are still mainly used to identify the causative pathogens in routine veterinary diagnosis. Because of the variation of phenotypic characteristics, the differentiation of Streptococcus or Streptococcus-like species solely based on biochemical reactions may result in misdiagnosis [2], and the genuine causative pathogen may be overlooked. This inaccurate data will give rise to improper disease control. In addition, the misidentification of S. suis may put the people working with pigs and pork at risk of the zoonotic infection without noticing it. Therefore, the appropriate identification method used in routine veterinary diagnosis is a crucial component for establishing the effective strategies for infectious disease control and prevention in both animals and humans.

In this study, in order to emphasize the usefulness of molecular techniques, such as PCR, for identification of Streptococcus species in routine veterinary diagnostic work-up, we collected the isolates from swine clinical specimens, which were

*Correspondence to: Takamatsu, D.: p1013dt@affrc.go.jp

O2019 The Japanese Society of Veterinary Science

This is an open-access article distributed under the terms of the Creative Commons Attribution Non-Commercial No Derivatives (by-nc-nd) License. (CC-BY-NC-ND 4.0: https://creativecommons.org/licenses/by-nc-nd/4.0/) 
Table 1. Primers used in this study

\begin{tabular}{llc}
\hline \multicolumn{1}{c}{ Primer name } & \multicolumn{1}{c}{ Sequence $\left(5^{\prime}\right.$-3') } & Reference \\
\hline $\begin{array}{l}\text { Species-specific PCR for } S \text {. suis } \\
\text { SSrecN-F }\end{array}$ & CTACAAACAGCTCTCTTCT & {$[9]$} \\
$\quad$ SSrecN-R & ACAACAGCCAATTCATGGCGTGATT & {$[9]$} \\
Amplification of partial $r e c N$ & & \\
recNF1 & CCTATCTGGTGAAACAGGTGCA & {$[24]$} \\
recNR2 & GTCCAGACACCCCTGTATCC & {$[24]$} \\
Sequencing of partial $r e c N$ & & \\
recNR2 & GTCCAGACACCCCTGTATCC & {$[24]$} \\
recNfs & GACCAGGAAGAATTGATGAA & {$[24]$} \\
recNfs2 & AACGGTCGATGATGTCTTGG & This study \\
recNrs2 & TCTGCTGCTTCAATCTCAGC & This study \\
Amplification of partial sodA & & \\
d1 & CCITAYICITAYGAYGCIYTIGARCC & {$[21]$} \\
d2 & ARRTARTAIGCRTGYTCCCAIACRTC & {$[21]$} \\
Sequencing of partial sodA & & \\
sodAfs & & This study \\
sodArs & TAAGCACCATGCGACTTATG & This study \\
\hline
\end{tabular}

biochemically identified as $S$. suis and Streptococcus species and verified the former identifications using molecular techniques.

Necropsy specimens from the lungs $(n=15)$, liver $(n=1)$, lymph nodes $(n=2)$, brain swabs $(n=3)$ and a vaginal swab $(n=1)$ of diseased pigs, as shown in Table S1, were submitted to the Microbiology Unit of the Kamphaengsaen Veterinary Diagnostic Laboratory, Kasetsart University during April 2014-March 2016. The brain swabs were swab samples of the meninges and brains of pigs collected using sterile cotton swabs. In total, 22 presumptive Streptococcus isolates, which were identified based on morphology and biochemical characteristics according to the routine bacterial identification procedures, were representatively selected for this study. Briefly, the colonies of pure culture on 5\% blood agar were selected for Gram staining and catalase testing. Streptococcus-suspected isolates were further tested for other biochemical and cultural characteristics including acid production from inulin, lactose, mannitol, raffinose, salicin, sorbitol and trehalose, esculin hydrolysis, hippurate hydrolysis and growth in $6.5 \%$ $\mathrm{NaCl}$. The results of the biochemical test panel were evaluated by referring to the previous information [15].

The DNA of bacterial cells was extracted using an E.Z.N.A. Bacterial DNA kit (Omega Bio-Tek, Doraville, GA, U.S.A.) following the manufacturer's instructions. Because S. suis is an important zoonotic pathogen and a reliable species-specific PCR method for authentic $S$. suis (recN PCR) has been developed recently [9], all presumptive Streptococcus isolates were primarily analyzed using the PCR according to the previous study [9]. The capsular polysaccharide synthesis gene (cps)-type of authentic $S$. suis isolates was additionally identified using two-step multiplex PCR targeting serotype-specific cps genes, as described previously [18]. QIAGEN Multiplex Master PCR Mix (Qiagen, Hilden, Germany) was used for multiplex PCR reactions according to the manufacturer's recommendations. The cps-types were numbered corresponding to the expected serotypes (e.g., serotype 3 to $c p s-$ type 3); however, because serotypes 2 and $1 / 2$ cannot be differentiated solely using this typing method, the isolates, which carried specific genes for serotypes 2 and 1/2, were further confirmed by co-agglutination tests using anti-serotype 1 and 2 sera following previous studies [6,12]. Furthermore, 16S rRNA gene sequences of the bacterial isolates were determined as described previously and analyzed using EzBioCloud (https://www.ezbiocloud.net/) [1,26].

The two housekeeping genes of an isolate, which showed the discordant identification results between the species-specific PCR and 16S rRNA gene sequencing, were further analyzed. A gene encoding recombination/repair protein (recN) and a gene encoding the manganese-dependent superoxide dismutase $(\operatorname{sod} A)$ were amplified and sequenced as described previously [24] with slight modification. The target regions were amplified using TaKaRa Ex Taq polymerase (Takara Bio Inc., Kusatsu, Japan) and the amplicons were purified using a QIAGEN PCR purification kit (Qiagen). Sequencing of PCR products was carried out using a $3130 x$ l DNA Analyzer (Applied Biosystems, Foster city, CA, U.S.A.). All primers used in this study are listed in Table 1. The sequences of recN and sodA (GenBank accession numbers MH329643 and MH329644, respectively) of our isolate were analyzed by comparing with those of $S$. suis serotype reference strains and $S$. suis strain P1/7. Accession numbers of sequences used in this analysis were described in a previous study [24]. The phylogenetic tree was further constructed using the neighbor-joining method with MEGA7 [11, 22].

According to the identification based on biochemical characteristics, 17 isolates from the lungs, brain swabs and tracheobronchial lymph node were identified as S. suis while one isolate from a vaginal swab was classified as Streptococcus dysgalactiae. Due to the limitation of traditional biochemical tests, the other four isolates were recognized as Streptococcus species. The results of the biochemical tests are shown in Table S2. All isolates were further examined using recN PCR [9]. Among the 17 presumptive $S$. suis isolates identified by biochemical tests, only five isolates gave positive recN PCR results, whereas three of the five presumptive non-S. suis isolates were also found to be recN-positive (Table 2). That is, among the 22 isolates from specimens of swine clinical cases, eight isolates were identified as $S$. suis by the species-specific PCR. These isolates showed various cps- 
Table 2. Sample sources and the results of bacterial identification

\begin{tabular}{|c|c|c|c|c|c|}
\hline \multirow[b]{2}{*}{$\begin{array}{l}\text { Strain } \\
\text { name }\end{array}$} & \multirow[b]{2}{*}{ Source } & \multicolumn{3}{|c|}{ Bacterial identification results based on } & \multirow[b]{2}{*}{$\begin{array}{c}\text { GenBank } \\
\text { accession number } \\
\text { of } 16 \mathrm{~S} \text { rRNA gene }\end{array}$} \\
\hline & & Biochemical characteristic $^{\text {a) }}$ & $\begin{array}{l}\text { Species-specific PCR for } \\
\text { Streptococcus suis (recN } \\
\text { PCR) and cps typing }\end{array}$ & $\begin{array}{l}\text { 16S rRNA gene sequencing } \\
\text { (\% similarity) }\end{array}$ & \\
\hline TRG1 & Lungb) & Streptococcus suis & Positive/cps-type 3 & Streptococcus suis $(99.8 \%)$ & MH329621 \\
\hline TRG2 & Lung b) & Streptococcus suis & Negative & Globicatella sanguinis $(99.73 \%)$ & MH329622 \\
\hline TRG3 & Lung b) & Streptococcus sp. & Negative & Streptococcus pluranimalium (99.59\%) & MH329623 \\
\hline TRG4 & $\begin{array}{l}\text { Mesenteric } \\
\text { lymph node }\end{array}$ & Streptococcus sp. & Negative & Streptococcus porcorum $(98.77 \%)$ & MH329624 \\
\hline TRG6 & Lung ${ }^{\text {b) }}$ & Streptococcus suis & Positive/cps-type 4 & Streptococcus suis $(99.86 \%)$ & MH329625 \\
\hline TRG7 & Lung b) & Streptococcus suis & Negative & Streptococcus pluranimalium $(99.59 \%)$ & MH329626 \\
\hline TRG8 & Vaginal swab ${ }^{\text {b) }}$ & Streptococcus dysgalactiae & Positive/cps-type 15 & Streptococcus suis $(98.71 \%)$ & MH329627 \\
\hline TRG10 & Lung & Streptococcus suis & Negative & $\begin{array}{l}\text { Streptococcus gallolyticus subsp. } \\
\text { gallolyticus }(99.8 \%)\end{array}$ & MH329628 \\
\hline TRG11 & Lung & Streptococcus suis & Negative & $\begin{array}{l}\text { Streptococcus gallolyticus subsp. } \\
\text { gallolyticus }(99.8 \%)\end{array}$ & MH329629 \\
\hline TRG12 & Liver & Streptococcus sp. & Positive/nontypable & Streptococcus suis $(99.18 \%)$ & MH329630 \\
\hline TRG14 & Lung ${ }^{b)}$ & Streptococcus sp. & Positive/cps-type 18 & Streptococcus suis $(99.8 \%)$ & MH329631 \\
\hline TRG15 & $\begin{array}{l}\text { Lung of aborted } \\
\left.\text { fetus }{ }^{b}\right)\end{array}$ & Streptococcus suis & Negative & Vagococcus fluvialis (100\%) & MH329632 \\
\hline TRG16 & Brain swab ${ }^{b}$ & Streptococcus suis & Negative & Streptococcus porcorum $(100 \%)$ & MH329633 \\
\hline TRG20 & Lung & Streptococcus suis & Negative & Globicatella sanguinis $(99.59 \%)$ & MH329634 \\
\hline TRG22 & Brain swab & Streptococcus suis & $\begin{array}{l}\text { Positive/cps-type } 2 \\
\text { (serotype } 2 \text { ) }\end{array}$ & Streptococcus suis $(99.73 \%)$ & MH329635 \\
\hline TRG24 & Lung ${ }^{b)}$ & Streptococcus suis & Positive/cps-type 21 & Streptococcus suis $(99.8 \%)$ & MH329636 \\
\hline TRG25 & Lung & Streptococcus suis & Negative & Aerococcus urinaeequi $(99.93 \%)$ & MH329637 \\
\hline TRG26 & Lung ${ }^{b)}$ & Streptococcus suis & Negative & Streptococcus hyovaginalis $(99.51 \%)$ & MH329638 \\
\hline TRG27 & Lung & Streptococcus suis & Negative & Streptococcus parasuis $(99.12 \%)$ & MH329639 \\
\hline TRG28 & $\begin{array}{l}\text { Tracheobronchial } \\
\text { lymph node }\end{array}$ & Streptococcus suis & Negative & Streptococcus suis $(99.05 \%)$ & MH329640 \\
\hline TRG29 & $\begin{array}{l}\text { Lung of aborted } \\
\text { fetus }\end{array}$ & Streptococcus suis & Negative & Globicatella sanguinis (99.73\%) & MH329641 \\
\hline TRG30 & Brain swab ${ }^{\text {b) }}$ & Streptococcus suis & $\begin{array}{l}\text { Positive/cps-type } 2 \\
\text { (serotype } 2 \text { ) }\end{array}$ & Streptococcus suis (99.8\%) & MH329642 \\
\hline
\end{tabular}

a) Biochemical characteristics of each strain are shown in Table S2. b) Co-infection was observed. The other bacterial species, which were found in the same specimen, are presented in Table S1.

types including cps-types 2, 3, 4, 15, 18,21, and cps-nontypable (Table 2), and some of these types including cps-type 2 (serotype 2) have been isolated from human cases [7]. In comparison with the results obtained from recN PCR, S. suis identification depending solely on biochemical characteristics exhibited $70.6 \%(12 / 17)$ false positive and $60 \%(3 / 5)$ false negative among isolates reevaluated in this study. Except for the isolate TRG28 from the tracheobronchial lymph node, the results of recN PCR correlated with those of 16S rRNA gene sequencing (Table 2). Although it is difficult to exactly distinguish between some species solely by $16 \mathrm{~S}$ rRNA gene sequences due to the high sequence identity, in addition to S. suis, other Streptococcus or Streptococcus-like species including Globicatella sanguinis $(n=2)$, Streptococcus pluranimalium $(n=2)$, Streptococcus gallolyticus subsp. gallolyticus $(n=2)$, Aerococcus urinaeequi $(n=1)$, Streptococcus hyovaginalis $(n=1)$ and Streptococcus parasuis $(n=1)$ were found to be isolated from the lungs of pigs with pulmonary diseases. G. sanguinis $(n=1)$ and Vagococcus fluvialis $(n=1)$ were found in lungs from aborted fetuses. Moreover, two Streptococcus porcorum isolates were recovered from a mesenteric lymph node and brain swab samples (Table 2).

Although TRG28 was identified as $S$. suis based on biochemical characteristics and the results from 16S rRNA gene sequencing, the recN PCR result was inconsistent with those results, suggesting that TRG28 was not an authentic S. suis (Table 2). Due to the discordant results, partial $r e c N$ and $\operatorname{sod} A$ sequences of TRG28 were further analyzed to determine the taxonomic position of this isolate because sequences of these two housekeeping genes showed a low similarity value at the species level in Streptococcus [5] and thus were utilized for classification of $S$. suis and $S$. suis-like species in a previous study [24]. Sequence comparison of the recN genes between TRG28 and $S$. suis strain P1/7 showed nucleotide sequence differences in the primer regions for recN PCR between the two strains (Fig. 1). These differences were considered to cause template-primer mismatches, resulting in the negative-recN PCR in TRG28. In the phylogenetic tree constructed based on the recN sequences, TRG28 was not included in the authentic $S$. suis clade (Fig. 2A). However, in the phylogenetic tree constructed based on sodA sequences, TRG28 was grouped with the authentic $S$. suis strains (Fig. 2B). These results suggested that TRG28 is a strain located on the border between $S$. suis and other species. According to the previous analyses of housekeeping genes in the $S$. suis lineage, six $S$. suis serotype reference strains (serotypes 20, 22, 26, 32, 33 and 34) were proposed to be taxonomically removed from $S$. suis [8, 24]. Furthermore, a previous 
$\mathrm{P} 1 / 7$

CTCTTTTCCTTTGAAAATAGCAGAGCTCTCGAGCAAATTTTGCTTGAACAGGGGATTGAA 60 CITTTTCCTTTGAAAATAGCAGAGCTCTGGAACAGATTTTGAGTGAACAGGGGATTGAA 60

P1/7 GTAGCTGACGAACTCATTATCCGCCGTGAAATCCTGCAAAATGGCCGTTCGGTCAGCCGT 120 TRG28 GTTGCGGACGAACTCATTATCCGTCGGGAAATCCTACAAAATGGCGTTCCGTGAGCCGT 120

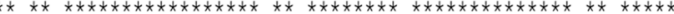

P1/7 GTCAATGGACAAATGGTCAATTTATCTGTCTTGAAACAGATTGGGCAATATTTAGTAGAT 180 TRG28 GTCAATGGGCAGATGGTTAATTTATCTGTCTTGAAACAAATTGGCCAGCACCTAGTGGAC 180

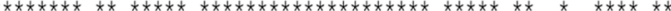

P1/7 ATTCATGGTCAACATGACCAGGAAGAATTGATGAAATCCCAGCACCATATCCGTCTTTTG 240 TRG28 ATCCACGGCCAGCATGACCAGGAAGAATTGATGAAGGCCCAGCACCATATCCGTCTTTTA 240 ** $* * * * *$ *

P1/7 GACAGTTTCGGAGAAGATGAATTTTGGAGTCTTAAAGACCGTTATCAGACAACCTTTGAT 300 TRG28 GACAGCTTTGGGGAAGATGAGTTTTGGGCTTTAAAAGAACGCTATCAGACCACTTTTGAT 300

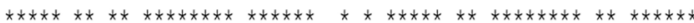

P1/7 GCCTATCGTAGTCTTCGCAAACGAGTTCTTGAAAAGCAAAAAAATGAGCAAGAACACAAG 360 TRG28 GCCTATCGTAGCCTCCGCAAACGAGTGCTCGAAAAGCAGAAAAATGAGCAGGAGCATAAG 360 $* * * * * * * * * * * * * * * * * * * * * * * * * * * * * * * * * * * * * * * * * * * * * * * * * * * * *$

P1/7 GCGCGGATTGAGATGCTAGAATACCAAATTGCTGAAATCGAAGCAGCGGATTTGAAGTCT 420 TRG28 GCTCGGATTGAGATGCTGGAATACCAAATTGCTGAGATTGAAGCAGCAGACCTGCAGTCT 420 ** $* * * * * * * * * * * * * * * * * * * * * * * * * * * * * * * * * * * * * * * * * * * * * * * * * * *$

P1/7 GGGGAAGATATTCAACTCAATCAGGAACGCGATAAACTGCTCAACCACAAACAAATTGCA 480 TRG28 GGTGAAGATGTTCAGCTCAATCAAGAGCGGGATAAGCTGCTCAACCACAAGCAGATTGCA 480

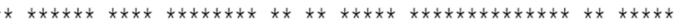

P1/7 GATACACTGACCAATGCCTATGCACTGTTAGACAATGAAGATTTTTCAAGCTTGAACAAC 540 TRG28 GATACCTTGACCAATGCCTATGCTCTGTTGGATAATGAAGATTTTTCAAGTTTGAATAAC 540 $* * * * * * * * * * * * * * * * * * * * * * * * * * * * * * * * * * * * * * * * * * * * * * * * * * * * * * * *$
P1/7 CTACGCTCAGCTATGAGTGACTTGCAAAGTCTGGAAGAATTTGATCCAGACTACAAACAG 600

TRG28 CTTCGTTCAGCCATGGGGGACTTGCAAAGCTTAGAAGAATTTGACCCAGAATACCAGCAG 600 $* * * * * * * * * * * * * * * * * * * * * * * * * * * * * * * * * * * * * * * * * * * * *$

P1/7 CTCTCTTCTAGCCTGACAGAAGCTTATTATGTCGTTGAAGATATAACCAAGCGTCTCAGC 660 TRG28 CTTTCTAGCAGTCTGACAGAGGCCTACTATGTTGTTGAAGATGTGACCAAGCGCCTCAGT 660

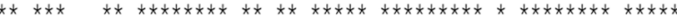

P1/7 GATGTGGTCGATAATCTAGATTTTGACGGCAATCGTCTCATGCAATTGGAAAGTCGTCTG 720 TRG28 GATGTGGTGGATAATCTAGACTTCGATGGCAATCGCTTAATGCAACTAGAAAGTCGGTTA 720

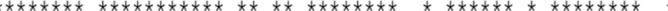

P1/7 GATTTGCTCAATACCATTACGAAGAAATATGGTGGAACTGTCGATGATGTTTTGGACTAT 780 TRG28 GATCTACTCCACACCATTACTAAAAAATACGGTGGAACGGTCGATGATGTCTTGGATTAT 780 $* * * * * * * * * * * * * * * * * * * * * * * * * * * * * * * * * * * * * * * * * * * * * * * * * * *$

P1/7 TTTAGTAAAATCAGCGAAGAATACAATCTATTGACAGGCAATGATTTATCTGGAGATGAT 840 TRG28 TTTGCTAAGATCAGCGAAGAATACAATCTCCTAACAGGAAATGACCTATCTGGAGATGAC 840

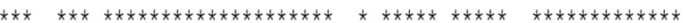

P1/7 TTGGAAGTTCAGCTTAAGAACTTAGAGAAAGAATTGGTTGAACGAGCAGGTCAGCTCAGC 900 TRG28 TTGGAAGTGCAACTCAAGACTCTGGAAAAAGAATTGGTGGGCTTAGCGACCGAACTCAGC 900 $* * * * * * * * * * * * * * * * * * * * * * * * * * * * * * * * * * * * * * * * * *$

P1/7 CAATCACGCCATGAATTGGCTGTTGTTTTAGAAGATATTATCCGCCAAGAATTGCAAGAC 960 TRG28 CAAGCCCGACATGAATTGGCAGTGGTCTTAGAAGACATTATCCGTCAGGAATTACAGGAC 960 ******************t*

P1/7 TTGTATATGGAGAAAGCTCGTTTCCAAGTTCGATTTACTAAAGGAAAATTTAATCGAGAA 1,020 TRG28 TTGTATATGGAGAAGGCGCGCTTCCAAGTTCGTTTTACCAAGGGCAAGTTTAACCGTGAG 1,020

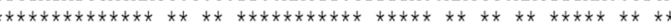

P1/7 GGAAATGAAACAGTAGAATTTTACATCTCTACCAAC 1,056

TRG28 GGCAATGAAACGGTGGAGTTTTACATCTCTACCAAC 1,056

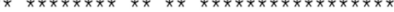

Fig. 1. Sequence alignment of partial recN between $S$. suis serotype 2 strain P1/7 and strain TRG28. Identical bases are indicated by asterisks. The primer regions of the species-specific PCR for S. suis (recN PCR) are highlighted with a gray background.

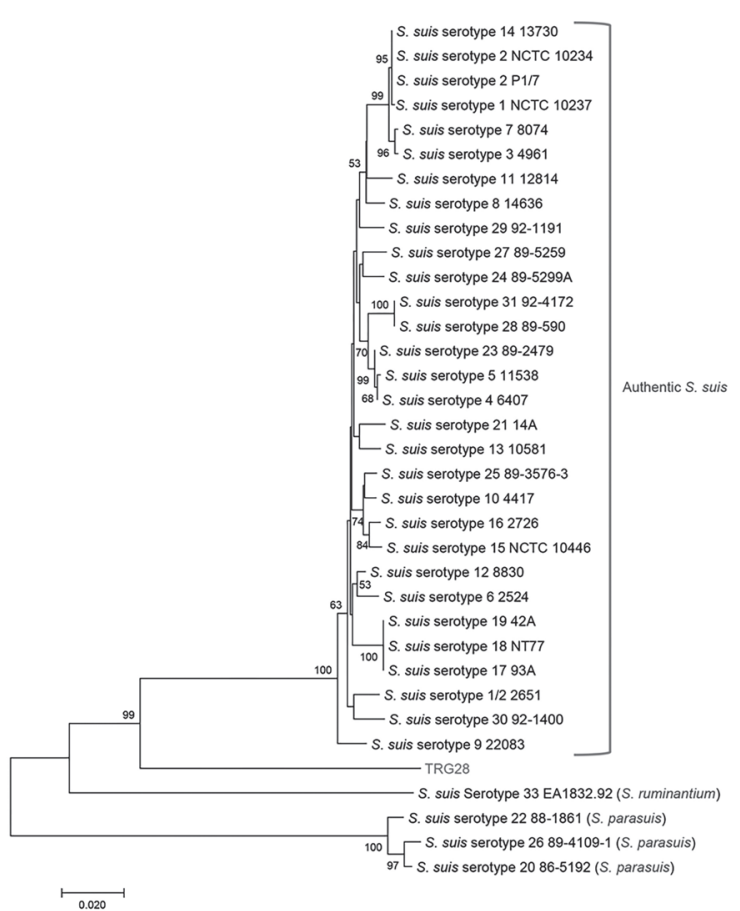

A

Fig. 2. Evolutionary history of $\operatorname{recN}(\mathrm{A})$ and $\operatorname{sod} A(\mathrm{~B})$ sequences inferred using the neighbor-joining method. Percentage ( $\geq 50 \%)$ of replicate trees in which the associated taxa clustered together in the bootstrap test (1,000 replicates) are shown next to the branches [4]. The tree is drawn to scale, with branch lengths in the same units as those of the evolutionary distances used to infer the phylogenetic tree. The evolutionary distances were computed using the p-distance method [17]. 
comparative genomic analysis [19] showed the lineage diversification in S. suis and suggested that S. suis serotypes 9, 13, 21, 24, 27, 29 and 31 should be considered divergent $S$. suis strains. TRG28 found in this study may also be one of the divergent $S$. suis strains.

The diversity of pathogens in swine lymph nodes and its impact for zoonotic infection have been indicated elsewhere [14]. In the current study, S. porcorum and the divergent S. suis (TRG28) were isolated from the enlarged mesenteric and tracheobronchial lymph nodes, respectively. The finding of these viable bacteria may account for the pathological changes of the lymph nodes. Another S. porcorum isolate was found accompanying Escherichia coli in a brain swab sample. Although $S$. porcorum was previously recovered from lesions of pneumonia and arthritis in pigs and was isolated from our clinical specimens, its pathogenicity could not be clearly concluded [25]. In addition, the other uncommon swine pathogens including G. sanguinis, S. pluranimalium and S. gallolyticus subsp. gallolyticus were notably isolated from lungs of pneumonia cases. As G. sanguinis is potentially considered as an emerging pathogen in humans [16] and may be related with animal infection, it is worthwhile to further develop the appropriate molecular diagnostic tool for investigating the infection of this pathogen.

In this study, we suggested that the misidentification of causative pathogens from clinical swine specimens in routine veterinary diagnosis could lead to the lack of awareness about the zoonotic disease transmission and subsequently increase the risk of outbreak. Moreover, underestimation of the unrecognized pathogens could potentially be a substantial obstacle to the prevention and management of infectious diseases in swine. Although matrix-assisted laser desorption/ionization time-of-flight mass spectrometry (MALDI-TOF MS) has been introduced to use as an accurate and rapid tool for bacterial identification including streptococcal identification [20], the application of the MALDI-TOF MS technique is still limited to some well-equipped laboratories. Taken all together, recN PCR is recommended for the identification of all presumptive Streptococcus species from clinical swine specimens.

ACKNOWLEDGMENTS. We thank Dr. Anusak Kerdsin for providing the DNA for positive control in the PCR reactions. This study was supported by the Thailand Research Fund (TRG5780267) and the Kasetsart University Research and Development Institute (KURDI), Bangkok, Thailand.

\section{REFERENCES}

1. Arai, R., Tominaga, K., Wu, M., Okura, M., Ito, K., Okamura, N., Onishi, H., Osaki, M., Sugimura, Y., Yoshiyama, M. and Takamatsu, D. 2012. Diversity of Melissococcus plutonius from honeybee larvae in Japan and experimental reproduction of European foulbrood with cultured atypical isolates. PLoS One 7: e33708. [Medline] [CrossRef]

2. Brigante, G., Luzzaro, F., Bettaccini, A., Lombardi, G., Meacci, F., Pini, B., Stefani, S. and Toniolo, A. 2006. Use of the Phoenix automated system for identification of Streptococcus and Enterococcus spp. J. Clin. Microbiol. 44: 3263-3267. [Medline] [CrossRef]

3. Facklam, R. and Elliott, J. A. 1995. Identification, classification, and clinical relevance of catalase-negative, gram-positive cocci, excluding the streptococci and enterococci. Clin. Microbiol. Rev. 8: 479-495. [Medline] [CrossRef]

4. Felsenstein, J. 1985. Confidence limits on phylogenies: an approach using the bootstrap. Evolution 39: 783-791. [Medline] [CrossRef]

5. Glazunova, O. O., Raoult, D. and Roux, V. 2010. Partial recN gene sequencing: a new tool for identification and phylogeny within the genus Streptococcus. Int. J. Syst. Evol. Microbiol. 60: 2140-2148. [Medline] [CrossRef]

6. Gottschalk, M., Higgins, R., Jacques, M., Mittal, K. R. and Henrichsen, J. 1989. Description of 14 new capsular types of Streptococcus suis. J. Clin. Microbiol. 27: 2633-2636. [Medline]

7. Goyette-Desjardins, G., Auger, J. P., Xu, J., Segura, M. and Gottschalk, M. 2014. Streptococcus suis, an important pig pathogen and emerging zoonotic agent-an update on the worldwide distribution based on serotyping and sequence typing. Emerg. Microbes Infect. 3: e45. [Medline] [CrossRef]

8. Hill, J. E., Gottschalk, M., Brousseau, R., Harel, J., Hemmingsen, S. M. and Goh, S. H. 2005. Biochemical analysis, cpn60 and 16S rDNA sequence data indicate that Streptococcus suis serotypes 32 and 34, isolated from pigs, are Streptococcus orisratti. Vet. Microbiol. 107: 63-69. [Medline] [CrossRef]

9. Ishida, S., Tien, L. H. T., Osawa, R., Tohya, M., Nomoto, R., Kawamura, Y., Takahashi, T., Kikuchi, N., Kikuchi, K. and Sekizaki, T. 2014. Development of an appropriate PCR system for the reclassification of Streptococcus suis. J. Microbiol. Methods 107: 66-70. [Medline] [CrossRef]

10. Köhler, W. 2007. The present state of species within the genera Streptococcus and Enterococcus. Int. J. Med. Microbiol. 297: 133-150. [Medline] [CrossRef]

11. Kumar, S., Stecher, G. and Tamura, K. 2016. MEGA7: Molecular evolutionary genetics analysis version 7.0 for bigger datasets. Mol. Biol. Evol. 33: 1870-1874. [Medline] [CrossRef]

12. Lakkitjaroen, N., Takamatsu, D., Okura, M., Sato, M., Osaki, M. and Sekizaki, T. 2011. Loss of capsule among Streptococcus suis isolates from porcine endocarditis and its biological significance. J. Med. Microbiol. 60: 1669-1676. [Medline] [CrossRef]

13. Lun, Z. R., Wang, Q. P., Chen, X. G., Li, A. X. and Zhu, X. Q. 2007. Streptococcus suis: an emerging zoonotic pathogen. Lancet Infect. Dis. 7: 201-209. [Medline] [CrossRef]

14. Mann, E., Dzieciol, M., Pinior, B., Neubauer, V., Metzler-Zebeli, B. U., Wagner, M. and Schmitz-Esser, S. 2015. High diversity of viable bacteria isolated from lymph nodes of slaughter pigs and its possible impacts for food safety. J. Appl. Microbiol. 119: 1420-1432. [Medline] [CrossRef]

15. Markey, B., Leonard, F., Archambault, M., Cullinane, A. and Maguire, D. 2013. The streptococci and related cocci. pp. 121-134. In: Clinical Veterinary Microbiology, 2nd ed. (Edwards, R. ed.), Mosby Elsevier, Maryland Heights.

16. Miller, A. O., Buckwalter, S. P., Henry, M. W., Wu, F., Maloney, K. F., Abraham, B. K., Hartman, B. J., Brause, B. D., Whittier, S., Walsh, T. J. and Schuetz, A. N. 2017. Globicatella sanguinis osteomyelitis and bacteremia: Review of an emerging human pathogen with an expanding spectrum of disease. Open Forum Infect. Dis. 4: ofw277. [Medline] [CrossRef]

17. Nei, M. and Kumar, S. 2000. Molecular Evolution and Phylogenetics, Oxford University Press, New York.

18. Okura, M., Lachance, C., Osaki, M., Sekizaki, T., Maruyama, F., Nozawa, T., Nakagawa, I., Hamada, S., Rossignol, C., Gottschalk, M. and 
Takamatsu, D. 2014. Development of a two-step multiplex PCR assay for typing of capsular polysaccharide synthesis gene clusters of Streptococcus suis. J. Clin. Microbiol. 52: 1714-1719. [Medline] [CrossRef]

19. Okura, M., Nozawa, T., Watanabe, T., Murase, K., Nakagawa, I., Takamatsu, D., Osaki, M., Sekizaki, T., Gottschalk, M., Hamada, S. and Maruyama, F. 2017. A locus encoding variable defence systems against invading DNA identified in Streptococcus suis. Genome Biol. Evol. 9: 1000-1012. [Medline] [CrossRef]

20. Pérez-Sancho, M., Vela, A. I., García-Seco, T., González, S., Domínguez, L. and Fernández-Garayzábal, J. F. 2017. Usefulness of MALDI-TOF MS as a diagnostic tool for the identification of Streptococcus species recovered from clinical specimens of pigs. PLoS One 12: e0170784. [Medline] [CrossRef]

21. Poyart, C., Quesne, G., Coulon, S., Berche, P. and Trieu-Cuot, P. 1998. Identification of streptococci to species level by sequencing the gene encoding the manganese-dependent superoxide dismutase. J. Clin. Microbiol. 36: 41-47. [Medline]

22. Saitou, N. and Nei, M. 1987. The neighbor-joining method: a new method for reconstructing phylogenetic trees. Mol. Biol. Evol. 4: 406-425. [Medline]

23. Segura, M. and Gottschalk, M. 2004. Extracellular virulence factors of streptococci associated with animal diseases. Front. Biosci. 9: 1157-1188. [Medline] [CrossRef]

24. Tien, L. H. T., Nishibori, T., Nishitani, Y., Nomoto, R. and Osawa, R. 2013. Reappraisal of the taxonomy of Streptococcus suis serotypes 20, 22, 26, and 33 based on DNA-DNA homology and $\operatorname{sodA}$ and recN phylogenies. Vet. Microbiol. 162: 842-849. [Medline] [CrossRef]

25. Vela, A. I., Sánchez, V., Mentaberre, G., Lavín, S., Domínguez, L. and Fernández-Garayzábal, J. F. 2011. Streptococcus porcorum sp. nov., isolated from domestic and wild pigs. Int. J. Syst. Evol. Microbiol. 61: 1585-1589. [Medline] [CrossRef]

26. Yoon, S. H., Ha, S. M., Kwon, S., Lim, J., Kim, Y., Seo, H. and Chun, J. 2017. Introducing EzBioCloud: a taxonomically united database of $16 \mathrm{~S}$ rRNA gene sequences and whole-genome assemblies. Int. J. Syst. Evol. Microbiol. 67: 1613-1617. [Medline] [CrossRef] 